\title{
Properties of supernovae in the early universe
}

\author{
Nozomu Tominaga* \\ Department of Physics, Faculty of Science and Engineering, Konan University, 8-9-1 Okamoto, \\ Kobe, Hyogo 658-8501, Japan \\ Kavli Institute for the Physics and Mathematics of the Universe, University of Tokyo, 5-1-5 \\ Kashiwanoha, Kashiwa, Chiba 277-8583, Japan \\ E-mail: tominaga@konan-u.ac.jp
}

The first metal enrichment in the universe was made by supernova ( $\mathrm{SN}$ ) explosions of population (Pop) III stars and the results are recorded in abundance patterns of extremely metal-poor (EMP) stars. We investigate the properties of Pop III SNe by comparing their nucleosynthetic yields with the abundance patterns of the EMP stars. Incresingly more EMP stars are now discovered. This allows us to statistically constrain their SN properties. We focus on the most metal-poor stars with $[\mathrm{Fe} / \mathrm{H}]<-3.5$ and present Pop III SN models reproducing well their individual abundance patterns. The distribution of the abundance patterns of EMP stars could be converted to that of SN properties, e.g., $M(\mathrm{Fe})$ and $M_{\text {rem }}$, in the early universe. Large samples of EMP stars, obtained by ongoing and planning EMP star surveys, e.g., SEGUE and Skymapper, would clarify distributions of properties of SNe in the early universe.

XII International Symposium on Nuclei in the Cosmos,

August 5-12, 2012

Cairns, Australia

${ }^{*}$ Speaker. 


\section{INTRODUCTION}

The universe was born without heavy elements and evolves chemically with metal enrichment driven by supernovae. The history of chemical evolution is recorded in the abundances of stars with low metallicities. The so-called metal-poor stars are low-mass stars formed in the early universe. Such stars preserve the information of elemental abundances in the early universe until the present days. Therefore, large programs surveying metal-poor stars provided substantial samples of metal-poor stars (e.g., HK survey: [1, 2]; Hamburg/ESO survey: [3]). Subsequent high-dispersion spectroscopic studies are determining the metallicities and abundances of the metal-poor stars. The metal-poor stars are classified by their metallicities as follows [4]: metal-poor stars (MP stars: $[\mathrm{Fe} / \mathrm{H}]<-1$ ), very metal-poor stars (VMP stars: $[\mathrm{Fe} / \mathrm{H}]<-2$ ), extremely metal-poor stars (EMP stars: $[\mathrm{Fe} / \mathrm{H}]<-3$ ), ultra metal-poor stars (UMP stars : $[\mathrm{Fe} / \mathrm{H}]<-4$ ), and hyper metal-poor stars (HMP stars: $[\mathrm{Fe} / \mathrm{H}]<-5$ ).

The first metal enrichment in the universe is due to massive stars and supernovae ( $\mathrm{SNe}$ ). The enrichment by a single $\mathrm{SN}$ dominates the preexisting metal contents in the early universe at metallicity (e.g., [5]). As the number of SNe increases with time, the remnants of $\mathrm{SNe}$ overlap with each other. The abundance of the universe becomes a superposition of numerous SNe and the universe evolves from unmixed to the well-mixed. Since the $r$-process elements show large scatters at $[\mathrm{Fe} / \mathrm{H}] \lesssim-2$, the transition between the unmixed and well-mixed universe is suggested to be at $[\mathrm{Fe} / \mathrm{H}] \sim-3 \cdots-2.5$ by hierarchical chemical evolution calculations (e.g., $[6,7])$.

Therefore, the properties of SNe in the early universe can be investigated by studying the abundance patterns of metal-poor stars with $[\mathrm{Fe} / \mathrm{H}]<-2.5$. The abundance patterns are reproduced by SN models [8-15]. Spherical SNe eject too much Fe. Thus the abundance patterns of the metalpoor stars and the chemical evolution are not reproduced (e.g., [16, 17]). Therefore, aspherical effects, i.e., the ejection of the inner matter and the fallback of the outer matter, are taken into account. Such an ejection and fallback process proposed by [8] called a mixing-fallback model. A Rayleigh-Taylor instability or an aspherical explosion is suggested as a physical process of the mixing-fallback model. Two-dimensional calculations have been performed by [18, 12, 14, 19, 20]. The abundance patterns of EMP stars are reproduced by these two-dimensional models but the Cenhanced metal-poor (CEMP) stars are not reproduced with the Rayleigh-Taylor instability.

In the recent years, the analysis of the stellar abundance has significantly improved. The first UMP star was reported in 2007 [21], and the most metal-deficient star was reported in 2012 [22]. Furthermore, the number of the metal-poor stars has increased dramatically e.g., by the Sloan Extension for Galactic Understanding and Exploration (SEGUE, [23]). Here, we focus on 18 most metal-poor stars with $[\mathrm{Fe} / \mathrm{H}]<-3.5^{1}$ and reproduce their abundance patterns with the mixingfallback model.

\section{Model \& Method}

We use progenitor models taken from $[10,11,13]$. First, we study three $M_{\mathrm{MS}}=25 M_{\odot}$ models. The calculation method and procedure are the same as [13].

\footnotetext{
${ }^{1}$ The stars are found in ADS (http://adswww.harvard.edu/) and SAGA database (http://saga.sci.hokudai.ac.jp/, [24]).
} 
We also take into account aspherical effects by applying the mixing-fallback model. The mixing-fallback model has three parameters characterizing the explosion: the initial mass cut $M_{\text {cut }}$, the outer boundary of the mixing region $M_{\text {mix }}$, and the ejection factor $f$ (see details in [13]). The physical process of the mixing-fallback model is an aspherical explosions, e.g., bipolar (jet-like) explosions $[18,12,14]$ and Rayleigh-Taylor instabilities [20]. We show that the mixing-fallback model well mimics the aspherical explosions except for entropy-sensitive elements like Sc, Ti, Cr, $\mathrm{Co}$, and $\mathrm{Zn}$ [14], and that the yields of aspherical explosions well reproduce the abundance patterns of the EMP stars $[18,12,14,20]$.

\section{Comparison with the observations}

In order to constrain properties of $\mathrm{SNe}$, it is necessary to determine abundances of many elements. In particular, the hydrodynamical features of $\mathrm{SNe}$ remain in the abundance ratios between elements synthesized in different layers and the thermodynamical features of $\mathrm{SNe}$ remain in the abundance ratios between elements synthesized in the same layer [14]. Thus, we select 18 metalpoor stars (including a binary) with $[\mathrm{Fe} / \mathrm{H}]<-3.5$ which have determined abundances or low upper limits of the following 4 elements: (1) one of $\mathrm{C}, \mathrm{N}$, and $\mathrm{O}$, (2) $\mathrm{Mg}$, (3) $\mathrm{Ca}$, and (4) Fe. The stars are 9 EMP stars, 5 CEMP stars, 2 UMP star, and 2 HMP stars (Figs. 1). The CEMP stars are CEMP-no stars that do not show an enhancement of $n$-capture elements. Since the adopted solar value is different depending on the papers, we recalculate the abundance ratios with the solar abundances proposed in [25].

All the EMP stars show high $[\mathrm{Co} / \mathrm{Fe}]$ and/or $[\mathrm{Zn} / \mathrm{Fe}](\gtrsim 0.1)$. These high ratios indicate highenergy explosions, i.e., hypernovae. Furthermore, all the EMP stars show $[\mathrm{Sc} / \mathrm{Fe}] \gtrsim 0$. The $[\mathrm{Sc} / \mathrm{Fe}]$ ratios are realized by the high-entropy explosions (e.g., [13]). Such a high-entropy and high-energy explosion with mixing and fallback is achieved only in jet-like explosions [14].

The origin of carbon enhancement in the CEMP-no stars is still under debate. We propose a faint $\mathrm{SN}$ characterized by a large amount of ${ }^{56} \mathrm{Ni}(\mathrm{Fe})$ fallback as the origin of the CEMP-no stars. The faint $\mathrm{SN}$ leads to high [C/Fe] because the fallback of carbon is less efficient than that of $\mathrm{Fe}$ [12]. Faint $\mathrm{SNe}$ are actually observed in the present days and characterized by low-energy explosions with low expansion velocities ( $E_{51}<1$, e.g., SN 1997D: [36]). Since the explosion energy is low, spherical fallback takes place. This is consistent with the observations of low $M\left({ }^{56} \mathrm{Ni}\right)$. On the other hand, a GRB without detection of an associated SN was also observed in 2006 (e.g., [37]). The upper limit on the amount of ${ }^{56} \mathrm{Ni}$ is as low as one hundredth of typical GRB-associated SNe. This implies the existence of a faint $\mathrm{SN}$ with relativistic jets.

Some CEMP-no stars show high [Co/Fe] and/or [Zn/Fe] ( 0): e.g., CS 29498-043 and HE 1300+0157 show high [Co/Fe], and CS 22949-037 shows high [Co/Fe] and [Zn/Fe]. Explosive nucleosynthesis in the high-entropy environment is required to reproduce high $[\mathrm{Co} / \mathrm{Fe}]$ and $[\mathrm{Zn} / \mathrm{Fe}]$. In the spherical symmetry, the high-entropy explosion is equivalent to the high-energy explosion in which the spherical fallback can not take place. This suggests that the CEMP-no stars formed from gases enriched by a faint $\mathrm{SN}$ with a relativistic jet. We note that the physical mechanism of the mixing-fallback model for the CEMP stars is a jet-like explosion with weak jets [12] because the mixing and fallback due to the Rayleigh-Taylor instability yields $[\mathrm{C} / \mathrm{Fe}] \sim+0.5[20]$. 


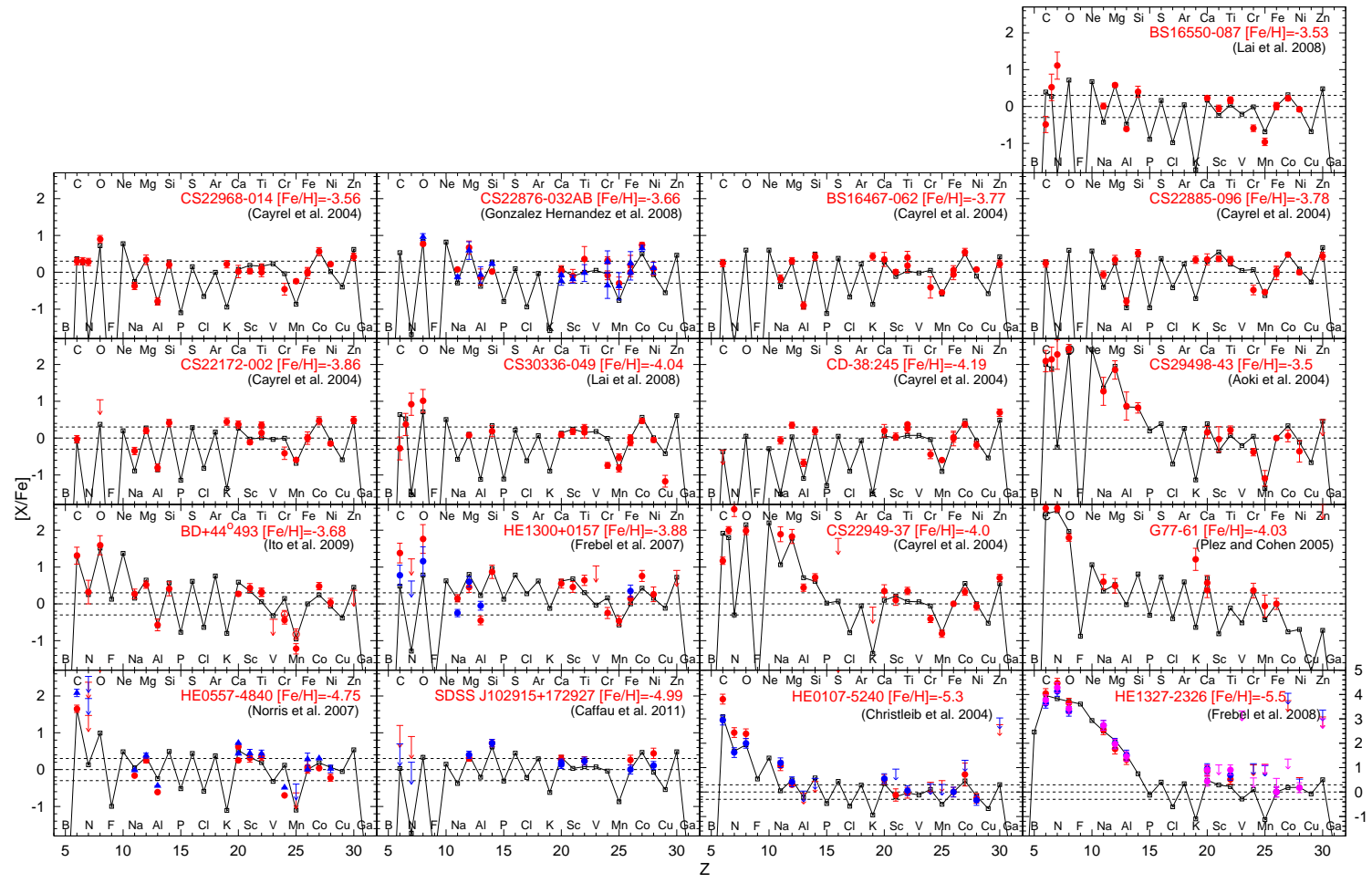

Figure 1: Abundance patterns of EMP stars (points) compared with yields of the SN models (lines). The names of stars and references are shown in the figure [26-31, 21, 32-35, 22].

Some stars show $[\mathrm{C} / \mathrm{N}](<0)$ and high $[\mathrm{N} / \mathrm{Fe}](>0)$. Stars with $[\mathrm{N} / \mathrm{Fe}]>+1$ are classified as $\mathrm{N}$-enhanced metal-poor (NEMP) stars (e.g., [38, 39]). Their carbon abundances have large scatter and $[\mathrm{N} / \mathrm{C}]$ ranges from $\sim-1$ to $\sim+1$. Several origins of $\mathrm{N}$ enhancement are proposed. Most of them display a signature of mixing in $\mathrm{Li}$ abundance and ${ }^{13} \mathrm{C} /{ }^{12} \mathrm{C}[38,39]$. The mixing enhances the conversion from carbon to nitrogen inside the NEMP stars. Such stars are called "mixed" stars $[38,39]$. For the mixed stars, we compare the models with the observations with the abundance of $\mathrm{C}+\mathrm{N}$, instead of $\mathrm{C}$ and $\mathrm{N}$. The other process is an enhanced mixing in progenitor star of parent $\mathrm{SN}$ due to rotational mixing and/or overshooting. However, the process can suppress $[\mathrm{C} / \mathrm{N}]$ only to $\sim-0.5[11,40]$. For example, since G77-61 is a dwarf, the N enhancement in G77-61 stems from the pre-enhancement in the SN progenitor.

Although there are 3 UMP stars with $[\mathrm{Fe} / \mathrm{H}]<-4$, we focus on 2 UMP stars with $[\mathrm{Fe} / \mathrm{H}]<$ -4.5 that were recently found: HE 0557-4840 with $[\mathrm{Fe} / \mathrm{H}]=-4.8$ [21] and SDSS J102915+172927 with $[\mathrm{Fe} / \mathrm{H}]=-4.7$ [22]. The abundance patterns of HE 0557-4840 and SDSS J102915+172927 are very similar to the CEMP and EMP stars, respectively, except for $[\mathrm{Fe} / \mathrm{H}]$. Therefore, we conclude that the parent SNe of HE 0557-4840 and SDSS J102915+172927 are similar to that of the CEMP and EMP stars, respectively, as well. The low $[\mathrm{Fe} / \mathrm{H}]$ could be the result of a wide distribution of $[\mathrm{Fe} / \mathrm{H}]$ (from $\sim-5$ to $\sim-1$ ) of a mixture of the $\mathrm{SN}$ ejecta and the pristine gases, according to 3-dimensional calculation of the expansion of SN ejecta [41, 42].

The first and second HMP stars were identified in 2002 and 2005: HE 0107-5240 $([\mathrm{Fe} / \mathrm{H}]=$ -5.2, [43]), and HE 1327-2326 ([Fe/H] $=-5.4$, [44]). The abundance patterns of HE1327- 
2326 [44] and HE0107-5240 [27, 45, 30] are quite unusual and shows high [C/Fe] $(\sim+4)$.

The mixing-fallback model successfully explains the abundance patterns of HMP stars $[9,11]$. Although they $[9,11]$ proposed that the abundance patterns of HMP stars are reproduced by a faint $\mathrm{SN}$ model with a low explosion energy, such a low-energy explosion cannot reproduce high $[\mathrm{Co} / \mathrm{Fe}]$ of HE 0107-5240 [45]. Therefore, assuming an jet-like explosion [12], we constructed an energetic explosion model with $E=5 \times 10^{51} \mathrm{erg}$ reproducing high [Co/Fe]. This can also enhance Sc [12]. In contrast to HE 0107-5240, Co is not detected in HE 1327-2326. Thus, the explosion energy of the parent SN of HE 1327-2326 is not constrained strictly. A high [Ti/Fe] ratio is suggestive of high entropy but the enhancement of $[\mathrm{Ti} / \mathrm{Fe}]$ is not enough to reproduce the observed value.

\section{Conclusion}

Many EMP stars have been observed. We focus on 18 EMP stars with $[\mathrm{Fe} / \mathrm{H}] \lesssim-3.5$ and show that the abundance patterns are well reproduced by SN models with different explosion properties. We propose that a large number of EMP stars combined with a large number of the elements observed in their abundance patterns can constrain the properties of Pop III SNe.

Although the abundance ratios of the EMP stars can be compared with the theoretical models, sophisticated 3-dimensional calculations taking into account the abundance distribution in the SN ejecta are required in order to interpret $[\mathrm{Fe} / \mathrm{H}]$ as well and constrain the next generation stars that can form from the enriched gas.

\section{Acknowledgments}

We thank the anonymous referee for language editing. Data analysis were in part carried out on the general-purpose PC farm at Center for Computational Astrophysics, CfCA, of National Astronomical Observatory of Japan.

\section{References}

[1] T. C. Beers, G. W. Preston, S. A. Shectman, AJ 103, 1987 (1992).

[2] T. C. Beers, S. Rossi, J. E. Norris, S. G. Ryan, T. Shefler, AJ 117, 981 (1999).

[3] N. Christlieb, Reviews in Modern Astronomy, R. E. Schielicke, ed. (2003), vol. 16 of Reviews in Modern Astronomy, pp. 191-+.

[4] T. C. Beers, N. Christlieb, ARA\&A 43, 531 (2005).

[5] J. Audouze, J. Silk, ApJ 451, L49 (1995).

[6] D. Argast, M. Samland, O. E. Gerhard, F. Thielemann, A\&A 356, 873 (2000).

[7] J. Tumlinson, ApJ 641, 1 (2006).

[8] H. Umeda, K. Nomoto, ApJ 565, 385 (2002).

[9] H. Umeda, K. Nomoto, Nature 422, 871 (2003).

[10] H. Umeda, K. Nomoto, ApJ 619, 427 (2005).

[11] N. Iwamoto, H. Umeda, N. Tominaga, K. Nomoto, K. Maeda, Science 309, 451 (2005). 
[12] N. Tominaga, et al., ApJ 657, L77 (2007).

[13] N. Tominaga, H. Umeda, K. Nomoto, ApJ 660, 516 (2007).

[14] N. Tominaga, ApJ 690, 526 (2009).

[15] A. Heger, S. E. Woosley, ApJ 724, 341 (2010).

[16] S. E. Woosley, T. A. Weaver, ApJS 101, 181 (1995).

[17] F. X. Timmes, S. E. Woosley, T. A. Weaver, ApJS 98, 617 (1995).

[18] K. Maeda, K. Nomoto, ApJ 598, 1163 (2003).

[19] C. C. Joggerst, S. E. Woosley, A. Heger, ApJ 693, 1780 (2009).

[20] C. C. Joggerst, et al., ApJ 709, 11 (2010).

[21] J. E. Norris, et al., ApJ 670, 774 (2007).

[22] E. Caffau, et al., Nature 477, 67 (2011).

[23] B. Yanny, et al., AJ 137, 4377 (2009).

[24] T. Suda, et al., PASJ 60, 1159 (2008).

[25] M. Asplund, N. Grevesse, A. J. Sauval, Cosmic Abundances as Records of Stellar Evolution and Nucleosynthesis, T. G. Barnes, III, F. N. Bash, eds. (2005), vol. 336 of Astronomical Society of the Pacific Conference Series, p. 25.

[26] R. Cayrel, et al., A\&A 416, 1117 (2004).

[27] N. Christlieb, et al., ApJ 603, 708 (2004).

[28] W. Aoki, et al., ApJ 608, 971 (2004).

[29] B. Plez, J. G. Cohen, $A \& A$ 434, 1117 (2005).

[30] R. Collet, M. Asplund, R. Trampedach, ApJ 644, L121 (2006).

[31] A. Frebel, et al., ApJ 658, 534 (2007).

[32] J. I. González Hernández, et al., A\&A 480, 233 (2008).

[33] D. K. Lai, et al., ApJ 681, 1524 (2008).

[34] A. Frebel, R. Collet, K. Eriksson, N. Christlieb, W. Aoki, ApJ 684, 588 (2008).

[35] H. Ito, W. Aoki, S. Honda, T. C. Beers, ApJ 698, L37 (2009).

[36] L. Zampieri, et al., MNRAS 338, 711 (2003).

[37] J. P. U. Fynbo, et al., Nature 444, 1047 (2006).

[38] M. Spite, et al., A\&A 430, 655 (2005).

[39] M. Spite, et al., A\&A 455, 291 (2006).

[40] G. Meynet, S. Ekström, A. Maeder, A\&A 447, 623 (2006).

[41] N. Nakasato, T. Shigeyama, ApJ 541, L59 (2000).

[42] J. S. Ritter, C. Safranek-Shrader, O. Gnat, M. Milosavljevic, V. Bromm, ArXiv e-prints (2012).

[43] N. Christlieb, et al., Nature 419, 904 (2002).

[44] A. Frebel, et al., Nature 434, 871 (2005).

[45] M. S. Bessell, N. Christlieb, From Lithium to Uranium: Elemental Tracers of Early Cosmic Evolution, V. Hill, P. François, \& F. Primas, ed. (2005), vol. 228 of IAU Symposium, pp. 237-238. 